Research Article

\title{
Scan Mirror Assembly for the Multispectral Scanning Polarimeter of Aerosol-UA Space Mission
}

\author{
Ivan Syniavskyi $\mathbb{D}^{1},{ }^{1}$ Yevgen Oberemok ${ }^{(D)},{ }^{2}$ Yuriy Ivanov, ${ }^{1}$ Mikhail Sosonkin, \\ Vladimir Kireyev, ${ }^{3}$ and Konstantin Akinin ${ }^{3}$ \\ ${ }^{1}$ Main Astronomical Observatory of the National Academy of Science of Ukraine, Kyiv, Ukraine \\ ${ }^{2}$ Taras Shevchenko National University of Kyiv, Kyiv, Ukraine \\ ${ }^{3}$ The Institute of Electrodynamics of the National Academy of Science of Ukraine, Kyiv, Ukraine
}

Correspondence should be addressed to Ivan Syniavskyi; syniavskyi@gmail.com

Received 1 September 2020; Revised 25 January 2021; Accepted 19 May 2021; Published 29 May 2021

Academic Editor: Wonho Jhe

Copyright (c) 2021 Ivan Syniavskyi et al. This is an open access article distributed under the Creative Commons Attribution License, which permits unrestricted use, distribution, and reproduction in any medium, provided the original work is properly cited.

\begin{abstract}
In this paper, the scan mirror assembly for the space experiment Aerosol-UA scanning polarimeter (ScanPol) is described. The aim of the Ukrainian space mission Aerosol-UA is to create a database of the optical characteristics of aerosol and cloud particles in the Earth's atmosphere over a long period of time. The optical characteristics of aerosol and cloud particles are derived from multiangular measurements. Multiangular scanning in ScanPol is provided by scan mirror assembly, which contains a reactive torque compensator electric motor and two scan mirrors, mounted on the shaft of the motor. The control system of the scan mirror assembly enables continuous scanning with a constant speed of the space under investigation. This control system tolerates movements of the orbiting satellite and preserves invariability of its spatial position. The polarimeter ScanPol is designed to acquire spatial, temporal, and spectral-polarimetric measurements simultaneously to minimize instrumental effects and "false" polarizations due to scene movement. Instrumental polarization, introduced by the mirrors of scan assembly, is minimized through the polarization compensated two-mirror scheme which contains two mirrors with orthogonal planes of incidence. In this paper, the polarimetric model of the polarization compensated two scan mirrors is considered. Theoretical calculations are given that substantiate the maximum allowable error of the relative angular position of the mirrors is $15 \operatorname{arcmin}\left(0.25^{\circ}\right)$, and the method of adjustment and control of the angular position of the mirrors is proposed. The polarization properties of mirrors are modelled in the spectral range of $370-1680 \mathrm{~nm}$ for bulk oxide-free aluminum. It is obtained that the maximum instrumental polarization of the unadjusted mirror system should be observed at $865 \mathrm{~nm}$, and thus, the polarization characteristics of the scanning system at a given wavelength could be considered as representative for ScanPol in general. The key steps for assembling the unit are illustrated.
\end{abstract}

\section{Introduction}

The impact of aerosol particles in the Earth's atmosphere on climate, by many estimates, can be comparable to the magnitude of the greenhouse gas effect. In the next few years, a number of polarimetric space experiments to study aerosols in the Earth's atmosphere are planned $[1,2]$.

The scientific space project Aerosol-UA has been proposed at the Main Astronomical Observatory of the National Academy of Sciences of Ukraine. The main goal is to measure detailed physical characteristics and estimate the chemical composition of natural and anthropogenic aerosols from a microsatellite with a multispectral scanning photometer-polarimeter (ScanPol) [3] and multispectral imaging polarimeter (MSIP) [4].

One of the important components of ScanPol is the scan mirror assembly (SMA), which allows multiangular scanning of sunlight that has been scattered by the underlying surface. ScanPol also contains the multispectral polarization state analyzer and the calibration units. The multispectral 
polarization state analyzer is designed for measurement and registration of photometric and polarization parameters of optical radiation in the spectral range of 370-1680 nm, while the calibration units maintain measurement accuracy during the mission. The operability of ScanPol is provided by the basic electronics module.

In this paper, the details of the design of the SMA of ScanPol are described. Characteristics of key elements of the SMA mirror system methodology of theoretical estimation of polarization parameters and the method for adjustment and control of the angular position of mirrors are described. The representational SMA is also shown. The ScanPol composition is shortly described in Section 2, characteristics of an electric motor with reactive torque compensator are given in Section 3, and two mirror systems are presented in Section 4 followed by Discussion and Summary in Sections 5 and 6 , respectively.

\section{Purpose and Composition}

The SMA of ScanPol consists of a polarization compensated two scan mirrors (TSM) (Figure 1) mounted on the shaft of electric motor (Figure 2). Rotating at a frequency of about $40 \mathrm{rpm}$, the TSM provides simultaneously the scanning of sunlight, scattered by the underlying surface in the range of angles $+50^{\circ}$ to $-60^{\circ}$ relative to the nadir and directing it to the input pupils of multispectral polarization state analyzer [5]. The size of the light zones of the mirrors provides the transmission of radiation with a beam diameter of at least $60 \mathrm{~mm}$, without vignetting (Figure 1).

The dimensional and mass characteristics of the SMA are as follows: light diameter of entrance and exit windows: $58 \mathrm{~mm}$; max diameter: $212 \mathrm{~mm}$; length: $142 \mathrm{~mm}$; and weight: $2.5 \mathrm{~kg}$.

\section{Electric Motor}

The electric motor provides continuous rotation of the TSM during the movement of the satellite in Earth orbit. The main requirement for the motor control system is to stabilize the speed of rotation and to keep the spatial position of the satellite unchanged. The operation of the electric motor can be divided into three main modes: acceleration of the rotor with inertial load to the required speed, rotation with a constant stabilized speed, and controlled braking.

According to Newton's third law, the torque generated by the motor is applied to both the motor rotor and the stator (reactive torque) and then to the satellite body on which the stator is mounted. The reactive torque rotates the satellite in the direction opposite to the rotation of the rotor with acceleration inversely proportional to the value of the corresponding axial moment of inertia of the satellite. It is likely that the reactive moment, especially in the dynamic mode of acceleration of the rotor, will exceed the moment created by the orientation system of the satellite, which will lead to an unacceptable change in its spatial position. The probability of such a phenomenon is the highest in satellites of nano-, mini-, and microclasses with limited capacities of orientation systems [6]. In order to avoid the negative

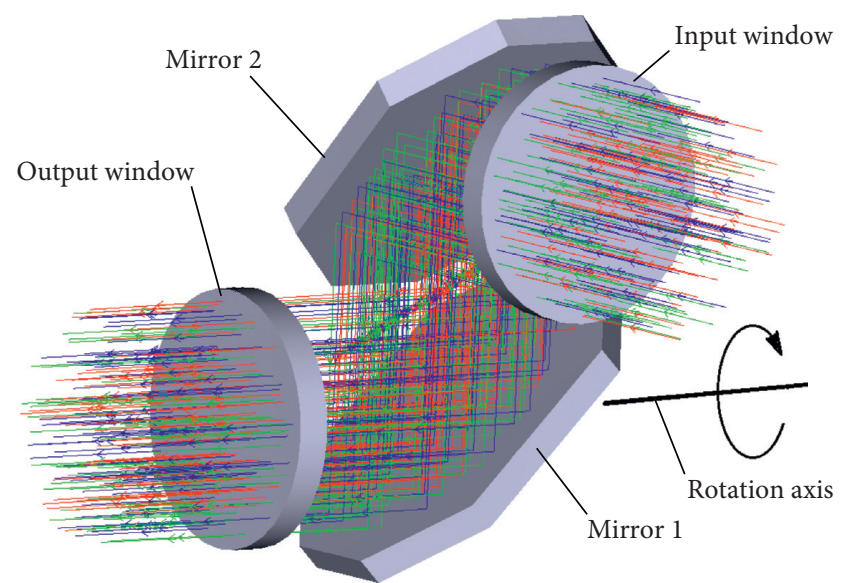

FIgURE 1: Optical layout of polarization compensated TSM.

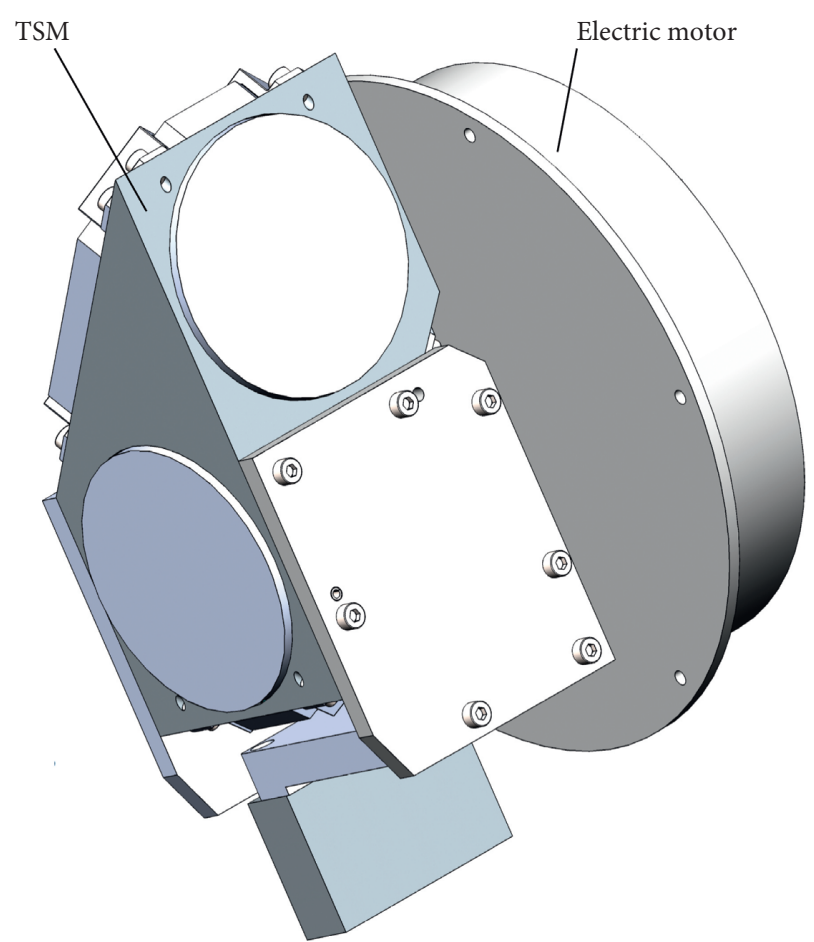

Figure 2: 3D model of the SMA.

consequences of the action of the reactive moment and not to add to the load the satellite orientation system, it is necessary to compensate the reactive moment created by the rotation motor.

The electric motor of the ScanPol was developed at the Institute of Electrodynamics of the National Academy of Sciences of Ukraine. This electromechanical system [7] consists of the main executive and secondary compensating motors and two corresponding control systems. The main and secondary electric motors are based on a brushless magnetoelectric disk-type machine with a slotless stator with surface-mounted rotor magnets. In the provided placement volume, the use of such a motor structure ensures the best energy, size, and operational characteristics of the electric motor and of the device as a whole [8]. Figure 3 schematically 


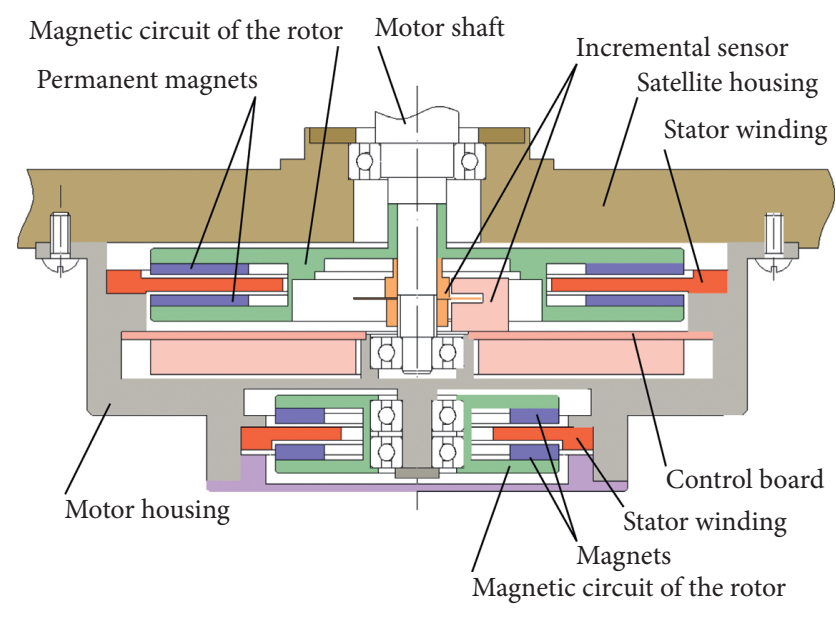

FIgURE 3: Electric motor of the scanning polarimeter.

shows the electric motor and indicates its main parts. The motor shaft of the main motor is connected to the magnetic circuit of the rotor, which houses the permanent magnets. The stator winding is placed in the space between the magnets, which is fixed on the motor housing. It is mounted on the satellite housing. The compensating motor has a similar structure-the magnetic circuit of the rotor with magnets and the stator winding. Between the main and compensating motors, the control board is mounted and the signal processing system of the incremental sensor mirror position is placed. The mirror position sensor consists of a metal disk mounted on the shaft with evenly spaced holes and a group of emitting LEDs and receivers on the control board. It also performs the function of synchronizing the ScanPol and MSIP [4]. A sequence of electrical pulses in the amount of 1000 pulses/rotation of the motor (after $0.36^{\circ}$ ) is formed on a separate signal channel at the motor output. The width of the zero pulse (reference point) is four times the width of the pulses that provide information about the angular position of the mirrors.

The main motor works in the stabilization mode of angular rotation speed. Thus, its start and braking are carried out smoothly with the given acceleration. Compensation for the reactive torque generated by the main motor is achieved by means of an additional motor mounted coaxially with the main motor, which is in idle mode and rotating in the opposite direction.

The motion of the rotating parts of both electric motors is described in its general form by the following equations:

$$
\begin{aligned}
\left(J_{1}+J_{m}\right) \frac{\mathrm{d} \omega_{1}}{\mathrm{~d} t} & =M_{D 1}=M_{1}-M_{F 1}, \\
J_{2} \frac{\mathrm{d} \omega_{2}}{\mathrm{~d} t} & =M_{D 2}=M_{2}-M_{F 2},
\end{aligned}
$$

where $\omega_{1}$ and $\omega_{2}$ are angular speeds of the rotors of the main and compensating motors, respectively; $J_{1}, J_{2}$, and $J_{m}$ are axial moments of inertia of the main and compensating rotors and the moment of inertia of the two-mirror unit, respectively; $M_{D 1}$ and $M_{D 2}$ are dynamic moments of the main and compensating motors, respectively; $M_{1}$ and $M_{2}$ are electromagnetic moments developed by main and compensating motors, respectively; and $M_{F 1}$ and $M_{F 2}$ are moments of friction of bearings of executive and compensating motors, respectively.

From (1), it follows that the compensation of the reactive torque of the main motors is provided under the condition of equality of the dynamic moments of the motors. The angular accelerations of the rotating parts must be inversely proportional to the ratio of their axial moments of inertia $e_{1} / e_{2}\left(J_{1}+J_{m}\right) / J_{2}$.

In the stabilization mode, when the main and compensating motors reach the required angular speeds, their angular acceleration tends to zero. The ratio between the angular speeds of the motors, which is the ratio of their moments of inertia $w_{2}=w_{1}\left(J_{1}+J_{m}\right) / J_{2}$, should be maintained.

The ability to vary the parameters of the compensating motor allows us to implement these conditions with acceptable weight and size. For example, for the considered electromechanical system, the relation was accepted as $\left(J_{1}+J_{m}\right) / J_{2}=25$, that is, the axial moment of inertia of the additional rotor is 25 times less than the magnitude of the moments of inertia of the rotor of the main motor with a two-mirror unit. Thus, the negative impact of the reactive torque of the main motor is achieved without a significant increase in mass and power consumption of the onboard equipment of the satellite.

In Table 1, the principal technical characteristics of main and compensating motors are presented.

\section{Two-Mirror System}

4.1. Requirements for the Mirror Alignment. Both mirrors of TSM (Figure 1) change the polarization of incident light. To minimize the instrumental light polarization of the TSM as a whole, a polarization compensated two-mirror scheme (Figure 4) is used. Note the instrumental polarization of an optical system is the polarization of light at the output of the optical system for an unpolarized input. The polarization compensation in scheme (Figure 4) is provided under the condition of the complete identity of optical characteristics of mirrors $M 1$ and $M 2$, when plane-parallel rays fall on mirrors $M 1$ and $M 2$ at the same angles and when the angle between the planes of incidence of rays at mirrors is $90^{\circ}$. When the aforementioned conditions are not met, the polarization compensation is violated too, and uncompensated instrumental polarization will be mixed into the polarization of the input scene. Nonparallelism of rays will also violate the polarization compensation of the mirror pair (Figure 4) [9].

For the evaluation of the influence of the accuracy of mirror alignment in the scheme of Figure 4 on the level of instrumental polarization of TSM, the Mueller matrix method was used.

In the Mueller method, the polarization of the beam is represented by the Stokes vector $\mathbf{S}$. The optical system is represented by the Mueller M matrix. A view of the Stokes vector $\mathbf{S}$ in the general form is given in the following equation: 
TABLE 1: The main technical characteristics of main and compensating motors.

\begin{tabular}{lcc}
\hline Parameter & Main motor & Compensating motor \\
\hline Angular speed $(\mathrm{rad} / \mathrm{s})$ & 4.484 & 112.1 \\
Torque coefficient $(\mathrm{Nm} / \mathrm{A})$ & 0.05408 & 0.017 \\
Moment of inertia $\left(\mathrm{kg} \cdot \mathrm{m}^{2}\right)$ & 0.002125 & 0.000085 \\
Active winding resistance $(\mathrm{Ohm})$ & 4.55 & 2.3 \\
Electromagnetic winding time constant (s) & 0.00032 & 0.00029 \\
Moment of resistance $(\mathrm{N} \mathrm{m})$ & 0.00132 & 0.000328 \\
Electromechanical time constant (s) & 2.204 & 0.4512 \\
Power consumption in operating mode (Watt) & 0.01 & 0.038 \\
\hline
\end{tabular}

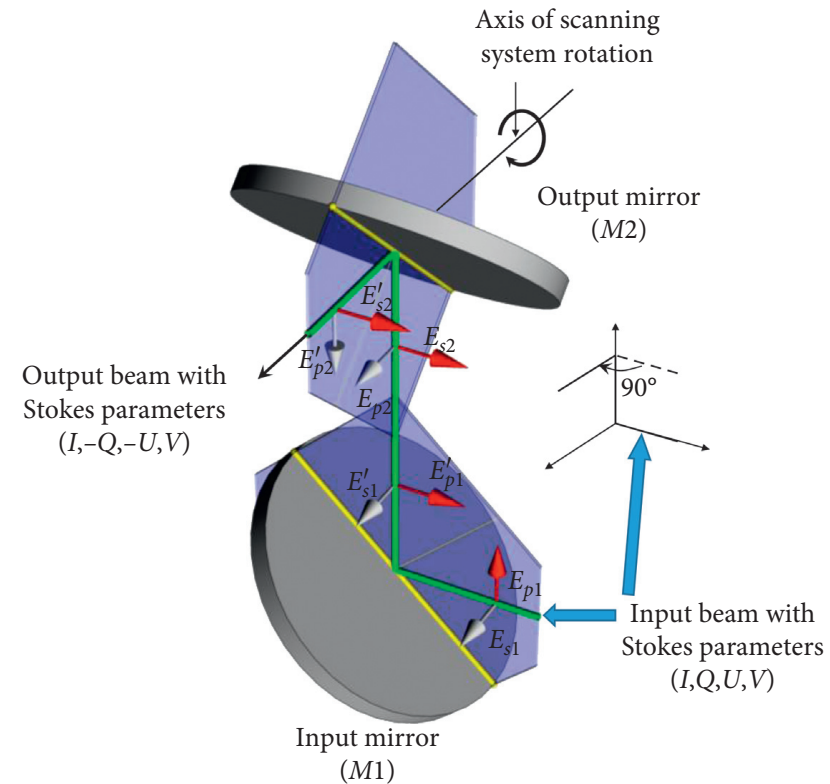

FIgURE 4: Polarization compensated two-mirror scheme.

$$
\begin{aligned}
& \mathbf{S}=\left[\begin{array}{l}
I \\
Q \\
U \\
V
\end{array}\right]=\left[\begin{array}{c}
I \\
I \cdot p \cdot \cos (2 \theta) \cos (2 \varepsilon) \\
I \cdot p \cdot \sin (2 \theta) \cos (2 \varepsilon) \\
I \cdot p \cdot \sin (2 \varepsilon)
\end{array}\right], \\
& p=\sqrt{\frac{Q^{2}+U^{2}+V^{2}}{I^{2}}},
\end{aligned}
$$

where $I, Q, U$, and $V$ are Stokes parameters; $I$ is also the total intensity of the light; $p$ is the degree of polarization of the light; and $\theta$ and $\varepsilon$ are the azimuth and angle of ellipticity of the light polarization ellipse, respectively.

Remote sensing often deals with partially or completely linearly polarized light. The angle of ellipticity of the partially or completely linearly polarized light is zero $\left(\varepsilon=0^{\circ}\right)$, and the fourth Stokes parameter is zero $(V=0)$ as well. Thus the Stokes vector takes the following form:

$$
\begin{aligned}
& \mathbf{S}=\left[\begin{array}{l}
I \\
Q \\
U \\
V
\end{array}\right]=\left[\begin{array}{c}
I \\
I \cdot p \cdot \cos (2 \theta) \\
I \cdot p \cdot \sin (2 \theta) \\
0
\end{array}\right], \\
& p=\operatorname{DoLP}=\sqrt{\frac{Q^{2}+U^{2}}{I^{2}}}, \\
& \theta=\operatorname{AoLP}=\frac{1}{2} \operatorname{arctg}\left(\frac{U}{Q}\right),
\end{aligned}
$$

where the parameter $p$ means the degree of linear polarization (DoLP) and $\theta$ is the azimuth of linear polarization (AoLP). The Mueller matrix $\mathbf{M}$ connects the light polarizations at the input and output of the optical system as

$$
\mathbf{S}^{\text {out }}=\mathbf{M} \cdot \mathbf{S}^{\text {in }} \text {, }
$$

where $\mathbf{S}^{\text {in }}$ and $\mathbf{S}^{\text {out }}$ are the Stokes vectors of the input and output light and $\mathbf{M}$ is the Mueller matrix of the optical system.

To describe the polarization characteristics of the TSM (Figures 1 and 4), it is necessary to define its Mueller matrix. The Mueller matrix of an ideal metal mirror is well known (see, for example, [10]): 


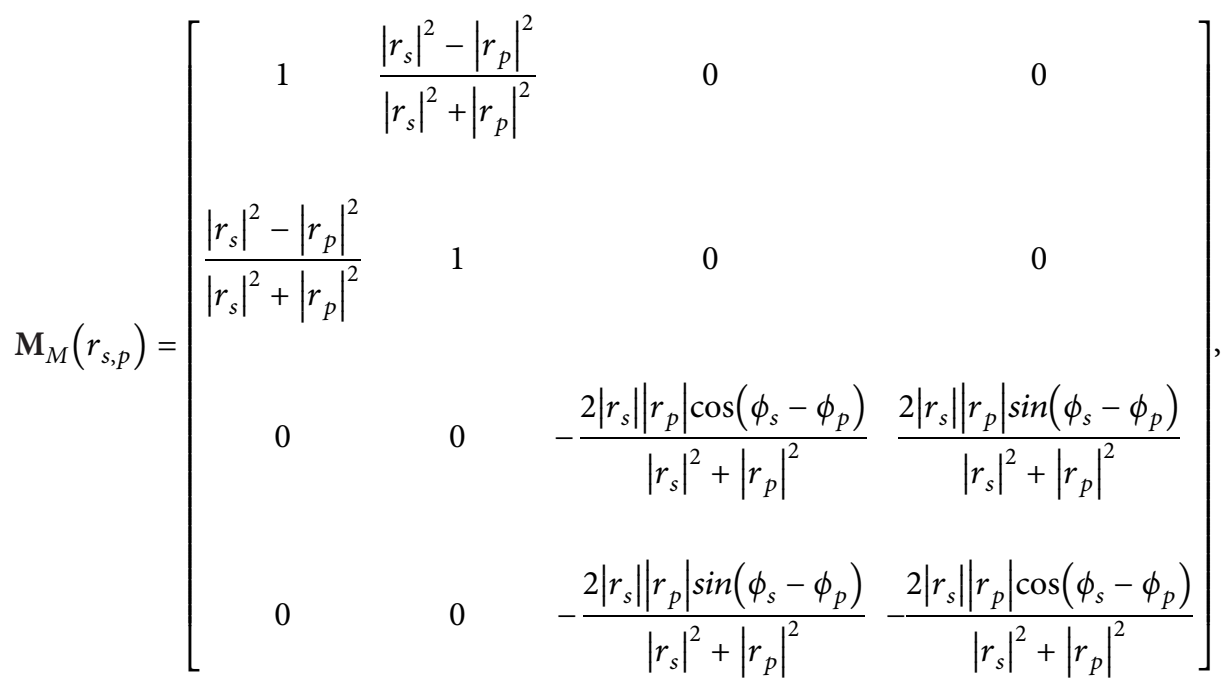

where $r_{s, p}=\left|r_{s, p}\right| \exp \left(\phi_{s, p}\right)$ are the complex reflection coefficients for the projection of the electric components of the incident beam on the plane of incidence $(p)$ and on its orthogonal plane $(s)$ (Figure 4$)$.

Note that matrix (5) is written in Eigen Geometry, where the $z$-axis is directed along the direction of light propagation and the $x$-and $y$-axes are parallel to the projections $E_{s}$ and $E_{p}$ electric component of the light, respectively. This coordinate system is the Eigen Geometry because the light with linear polarizations along the $x$ - or $y$-axis of this coordinate system does not change its polarization when reflected by the mirror. When rotating the coordinate system of the incident light around the $z$-axis by an angle $\alpha$, matrix (5) will turn to

$$
\mathbf{M}_{M}\left(r_{s, p}, \alpha\right)=\mathbf{M}_{R}(-\alpha) \mathbf{M}_{M}\left(r_{s, p}\right) \mathbf{M}_{R}(\alpha),
$$

where $\mathbf{M}_{R}(\alpha)$ is the matrix of rotation:

$$
\mathbf{M}_{R}(\alpha)=\left[\begin{array}{cccc}
1 & 0 & 0 & 0 \\
0 & \cos (2 \alpha) & \sin (2 \alpha) & 0 \\
0 & -\sin (2 \alpha) & \cos (2 \alpha) & 0 \\
0 & 0 & 0 & 1
\end{array}\right] .
$$

In the normal position, the planes of incidence of the rays on the mirrors $M 1$ and $M 2$ (Figure 4) are rotated relative to each other by an angle of $90^{\circ}$, so for the mirror $M 2$ $-\alpha=90^{\circ}$. According to the Muller matrix method, taking into account equations (5)-(7), the polarization characteristics of TSM can be described by a matrix product of the form:

$$
\mathbf{M}_{\mathrm{TSM}}=\mathbf{M}_{M 2}\left(r_{s, p}, 90^{\circ}\right) \cdot \mathbf{M}_{M 1}\left(r_{s, p}, 0^{\circ}\right),
$$

where $\mathbf{M}_{\mathrm{TSM}}$ is the general Muller matrix for two scan mirrors, $\mathbf{M}_{M 1}\left(r_{s, p}^{0^{\circ}}\right)$ is the Muller matrix of the mirror $M 1$, and $\mathbf{M}_{M 2}\left(r_{s, p}, 90^{\circ}\right)$ is the matrix of the mirror $M 2$, rotated by $90^{\circ}$ with respect to the first one, which is calculated using (5).

Assuming that the polarization characteristics $r_{s, p}$ of the first and the second mirrors of the configuration (Figure 4) are identical, from (8) it could be obtained that $\mathbf{M}_{\mathrm{TSM}}$ is an identity diagonal matrix with some scale factor. In other words, a pair of mirrors (Figure 4) in this case will be polarization-neutral (i.e., polarization compensated). The misalignments of the system mirrors will affect the polarization compensation. For the sake of simplicity, let fix the mirror $M 1$ and assume that the light falls on it at the angle $i=45^{\circ}$. The displacement of the second mirror relative to the exact position comes down to the rotations relative to two axes: "Axis 1" and "Axis 2" (Figure 5). This simplification is obviously acceptable because the degree of neutrality of the mirror system (Figure 4) will depend on the difference between the angles of the light incidence on the mirrors $M 1$ and $M 2$ and on the angle $\alpha$ between their planes of incidence.

The tilts of the mirror $M 2$ around axis 1 (Figure 5) correspond to the change of the angle $\alpha$ by the value of $\beta$ relative to exact position $\alpha=90^{\circ}$. The tilts of the mirror $M 2$ around axis 2 (Figure 5) correspond to the change of the incidence angle by the value of $\gamma$ relative to exact position $i=45^{\circ}$. The dependence of the mirror system matrix on the tilts of the mirror $M 2$ can be determined using (8) as

$$
\mathbf{M}_{\mathrm{TSM}}^{\prime}\left(r_{s, p}^{\prime}, \beta\right)=\mathbf{M}_{M 2}\left(r_{s, p}^{\prime}, 90^{\circ}+\beta\right) \cdot \mathbf{M}_{M 1}\left(r_{s, p}, 0^{\circ}\right),
$$

where $r_{s, p}^{\prime}$ are the new complex reflection coefficients of the mirror $M 2$ with some tilt $\gamma$ around axis 2 (Figure 5).

The coefficients of reflection $r_{s, p}$ are related to the angle of incidence and the complex refractive index of the mirror material $N(\lambda)=n(\lambda)-j \cdot k(\lambda)$ through Fresnel and Snell's equations:

$$
\begin{aligned}
r_{s} & =\frac{N_{0} \cos (i)-N(\lambda) \cos (\varphi)}{N_{0} \cos (i)+N(\lambda) \cos (\varphi)}, \\
r_{p} & =\frac{N(\lambda) \cos (i)-N_{0} \cos (\varphi)}{N(\lambda) \cos (i)+N_{0} \cos (\varphi)}, \\
N_{0} \sin (i) & =N(\lambda) \sin (\varphi),
\end{aligned}
$$

where $\lambda$ is the light wavelength, $j$ is the imaginary unit, $N_{0}=1$ is the refractive index of air, and $\varphi$ is the angle of refraction.

As it was assumed above, the light incidence angle for the mirror $M 1$ is $i=45^{\circ}$. Since for the mirror $M 2$ this angle is 


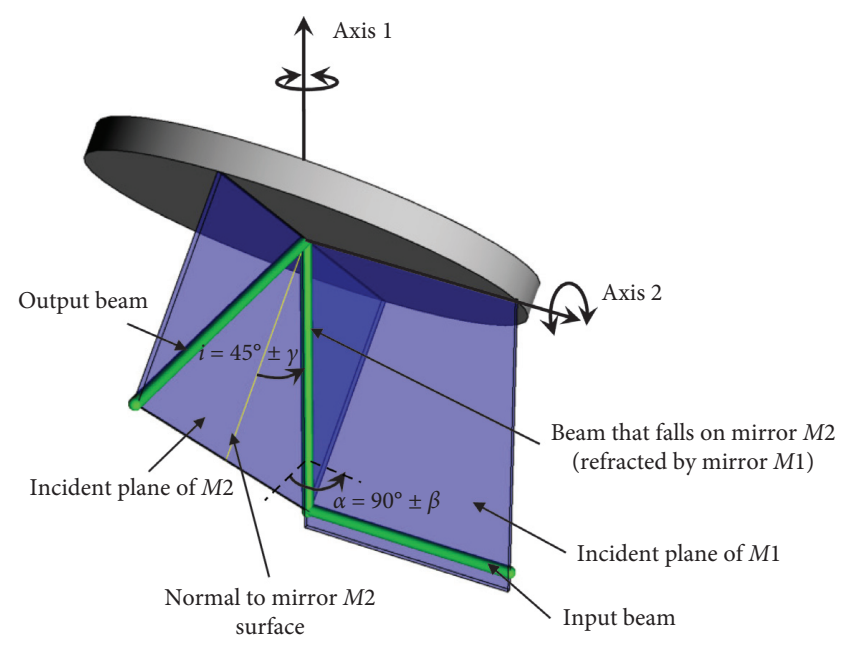

FIGURE 5: The main axes of the displacement of the mirror M2 of TSM.

changed to $i=45^{\circ} \pm \gamma$, according to (10) and (11), the reflection coefficients $r_{s, p}^{\prime}$ are not equal to $r_{s, p}$ for $M 1$.

In the SMA, aluminum mirrors are used. To calculate $r_{s, p}^{\prime}$ in (10) and (11), it is necessary to substitute the refractive index $N(\lambda)$ for aluminum. It is well known that the accurate polarization properties of aluminum mirrors are difficult to model. In fact, the refractive index of aluminum mirror strongly depends on (1) the coating process features [11], (2) the presence of a film of alumina $\left(\mathrm{Al}_{2} \mathrm{O}_{3}\right)$ on the mirror, and (3) the presence of dust on the surface [10]. Therefore, the information on the dispersion dependences of aluminum films differs in different references (see, for example, [11-15]). Our paper uses data from [16] based on [11]. Table 2 and Figure 6 show the real $n(\lambda)$ and imaginary $k(\lambda)$ part of the complex refractive index $N(\lambda)$ of the model aluminum mirror for the wavelengths used in ScanPol.

Using the data from Table 2 and equations (4) and (9)-(11) and considering the tilts of the mirror M2 around axes 1 and 2 in the ranges of angles $\beta, \gamma \in\left[-1^{\circ}, 1^{\circ}\right]$ relative to exact positions $\alpha=90^{\circ}$ and $i=45^{\circ}$, the boundary estimates were obtained for the degree $p_{\text {inst }}=\max \left|p_{0}-p^{\prime}\right| 100 \%$ (Figure 7) and the azimuth of the instrumental polarization $\theta_{\text {inst }}=\max \left|\theta_{0}-\theta^{\prime}\right|$ (Figure 8 ), where $p_{0}$ and $\theta_{0}$ are, respectively, the degree and azimuth of the linear polarization of the light at the input of the TSM and $p^{\prime}$ and $\theta^{\prime}$ are, respectively, the degree and azimuth of the linear polarization of the light at the output of the TSM.

The $p_{\text {inst }}$ does not depend on $p_{0}$, but it is increased with an increase in $\beta$ and $\gamma$ (see Figure 8). The $\theta_{\text {inst }}$ dependence with $\beta$ and $\gamma$ is increased as the value of $p_{0}$ decreases from 1 to 0 (see Figures 8 and 9). Figure 8 shows the $\theta_{\text {inst }}$ estimates at $p_{0}=0.2$.

Figures 7-9 show the significant dependence of the TSM instrumental polarization on the light wavelength. It is seen the value of instrumental polarization is the greatest for the spectral range $\lambda=865 \mathrm{~nm}$. Thus, in setting accuracy requirements for the alignment of the mirror system, we relied on the spectral component $\lambda=865 \mathrm{~nm}$.
TABLE 2: Real and imaginary parts of complex refractive index for aluminum mirrors.

\begin{tabular}{lcc}
\hline$\lambda(\mathrm{nm})$ & $n$ & $k$ \\
\hline 370 & 0.31760 & 3.8892 \\
410 & 0.39609 & 4.3368 \\
555 & 0.80835 & 5.9053 \\
865 & 2.0313 & 7.1846 \\
1378 & 1.1581 & 12.489 \\
1620 & 1.4214 & 14.706 \\
\hline
\end{tabular}

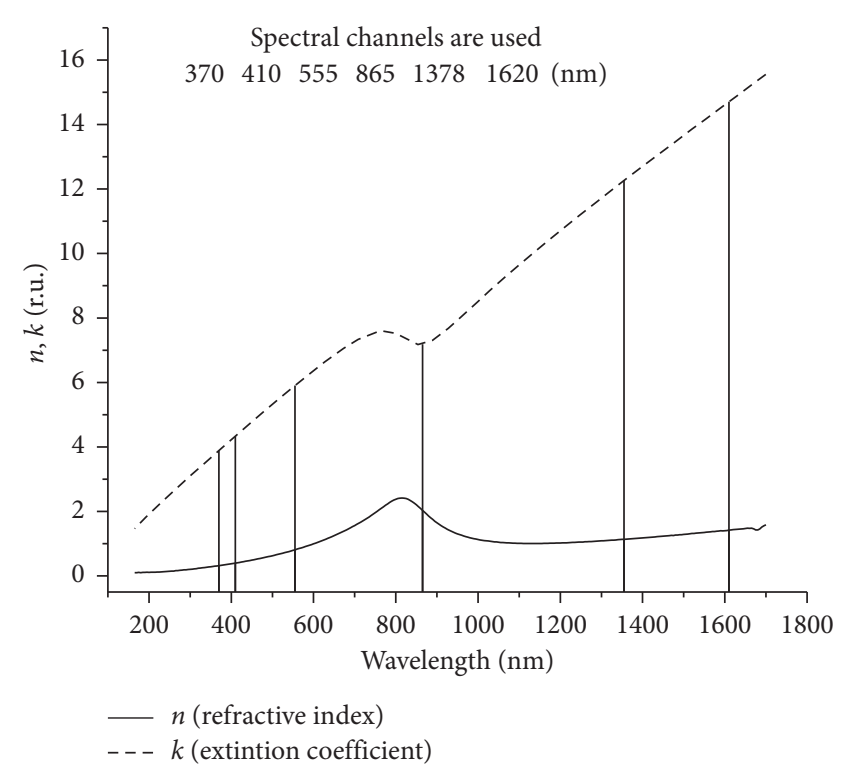

FIGURE 6: Dispersion of real and imaginary parts of complex refractive index of a thick layer of aluminum (bulk).

The declared maximum error in determining the degree of linear polarization of light by ScanPol in all spectral ranges is $\Delta p \leq 0.15 \%$. The declared maximum error in determining the azimuth of the linear polarization is $\Delta \theta \leq 0.2^{\circ}$ at $p_{0} \geq 0.2$ [17] since here $\Delta \theta$ has a weak dependence on $p_{0}$. As it was expected for $p_{0}>0.2$ the dependence of $\Delta \theta$ on $p_{0}$ will grow significantly, thereby under this condition there is no sense to determine $\theta$ separately due to the significant error. The expectation has been confirmed at least for the spectral component $\lambda=865 \mathrm{~nm}$ (Figure 9).

Given the other sources of systematic errors in ScanPol to achieve the declared error limits $\Delta p$ and $\Delta \theta$, the instrumental polarization of the TSM should not exceed

$$
\begin{aligned}
& p_{\text {inst }} \leq \frac{\Delta p}{2} \approx 0.08 \%, \\
& \theta_{\text {inst }} \leq \frac{\Delta \theta}{2} \approx 0.1^{\circ} .
\end{aligned}
$$

From the dependences in Figures 7-9 for the spectral component $\lambda=865 \mathrm{~nm}$, it can be seen that (12) will be met if the errors of the mirror setup (Figure 5) do not exceed $0.25^{\circ}$ (15 arcmin) 


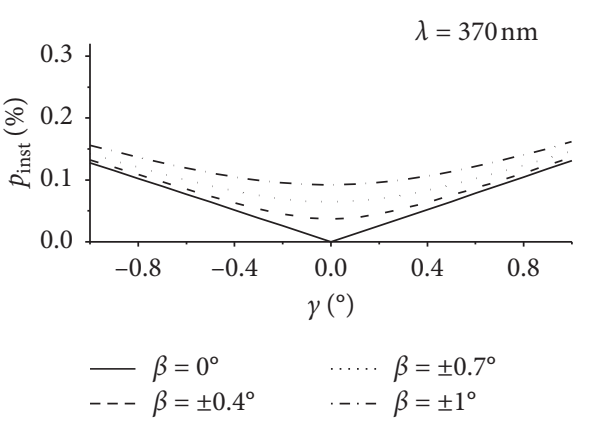

(a)

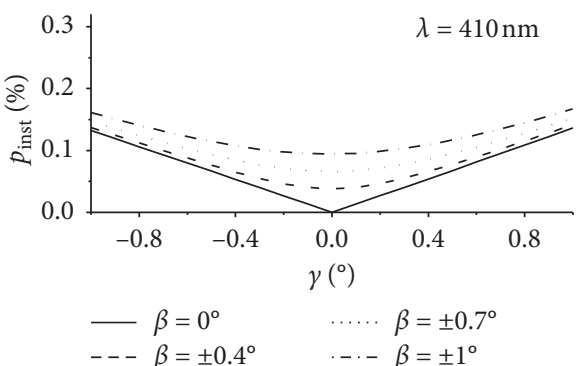

(c)

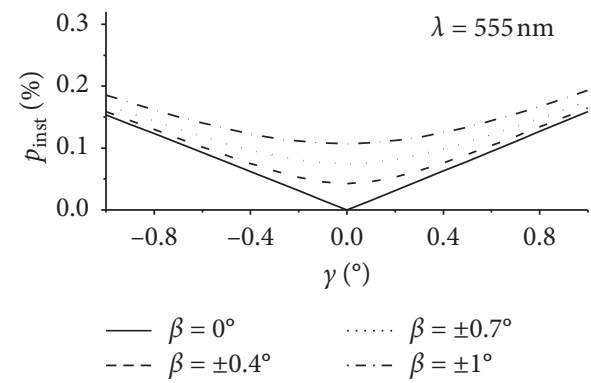

(e)

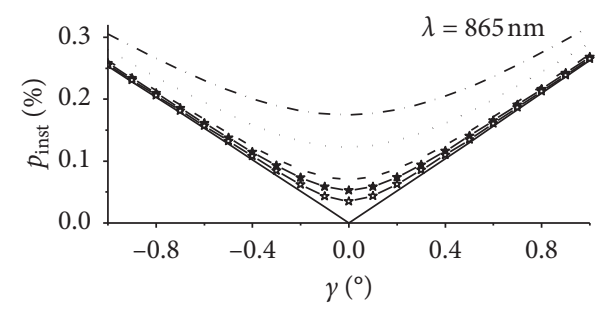

$$
\begin{aligned}
& -\beta=0^{\circ} \quad--\beta= \pm 0.4^{\circ} \\
& -\star-\beta= \pm 0.2^{\circ} \quad \cdots \cdots, \beta= \pm 0.7^{\circ} \\
& -\star-\beta= \pm 0.3^{\circ} \quad--\beta= \pm 1^{\circ}
\end{aligned}
$$

(b)

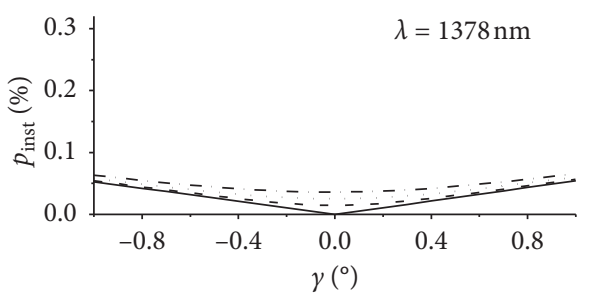

$$
\begin{aligned}
& -\beta=0^{\circ} \quad \cdots \cdots= \pm 0.7^{\circ} \\
& --\beta= \pm 0.4^{\circ} \quad--\beta= \pm 1^{\circ}
\end{aligned}
$$

(d)

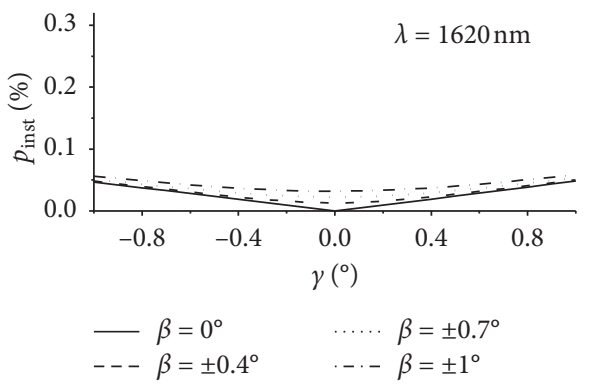

(f)

Figure 7: The dependence of the boundary value of the degree $p_{\text {inst }}$ of the instrumental polarization of the TSM on the mirror $M 2$ tilt around axes 1 and 2 (as shown in Figure 5).

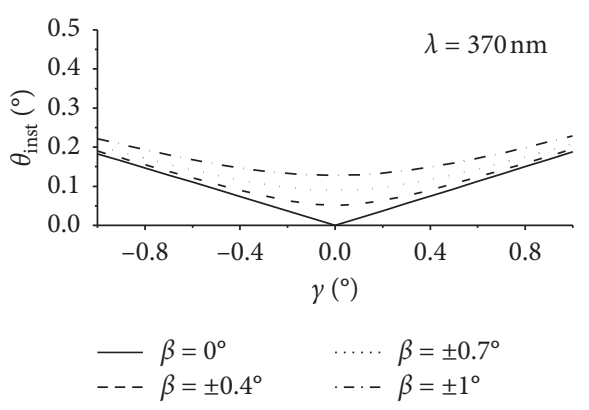

(a)

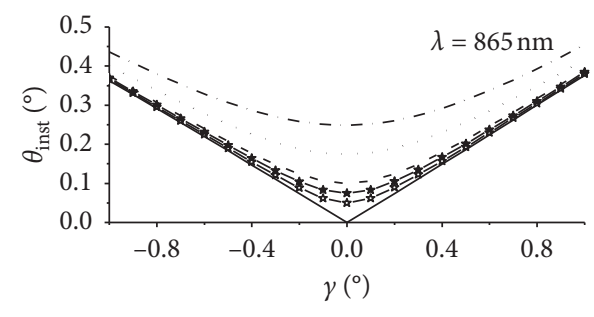

$$
\begin{aligned}
-\beta & =0^{\circ} \\
-\star-\beta & = \pm 0.2^{\circ} \\
\text { - } \beta & = \pm 0.3^{\circ}
\end{aligned}
$$$$
--\beta= \pm 0.4^{\circ}
$$$$
\text { … } \beta= \pm 0.7^{\circ}
$$$$
\text { -. } \beta= \pm 1^{\circ}
$$

(b)

Figure 8: Continued. 


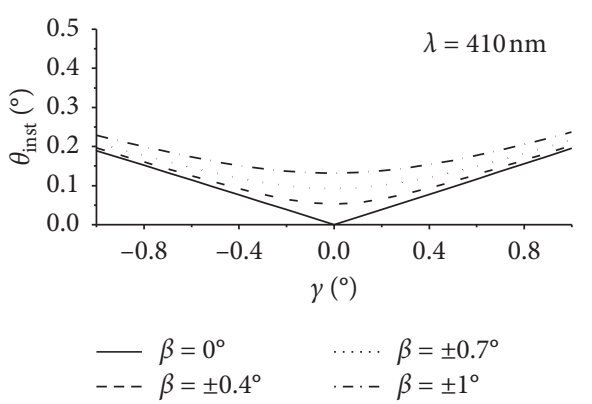

(c)

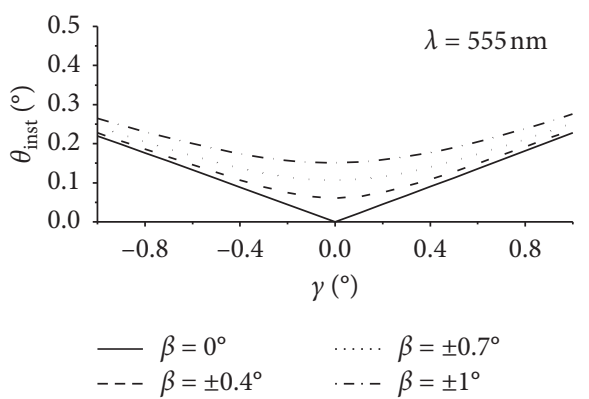

(e)

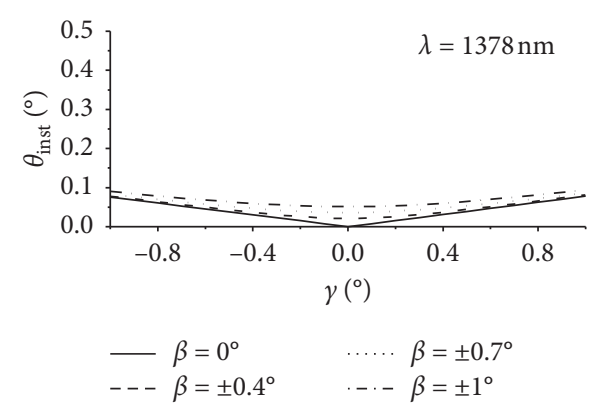

(d)

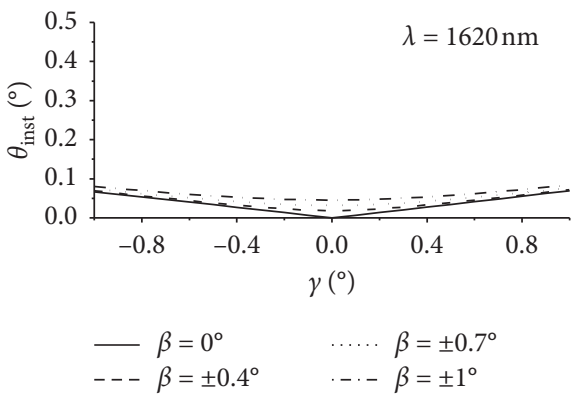

(f)

Figure 8: Dependence of the extremum value of azimuth $\theta_{\text {inst }}$ of the instrumental polarization of the TSM on the mirror $M 2$ tilt around axes 1 and 2 (as shown in Figure 5) at degrees of linear polarization $p_{0}=0.2$.
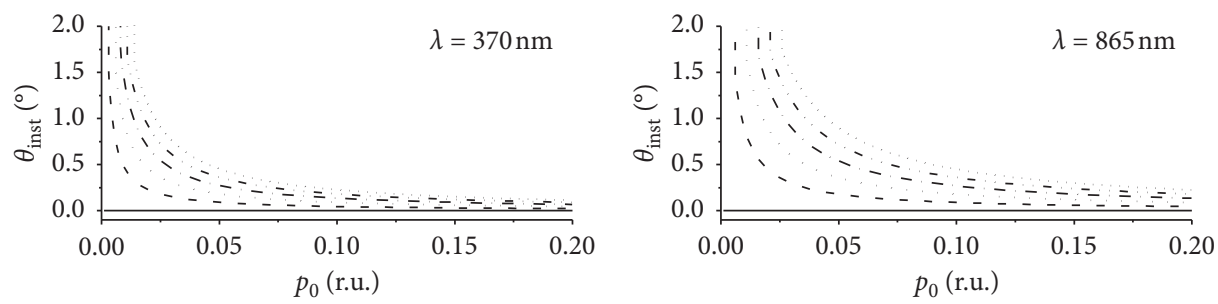

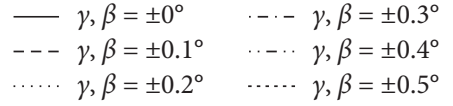

(a)

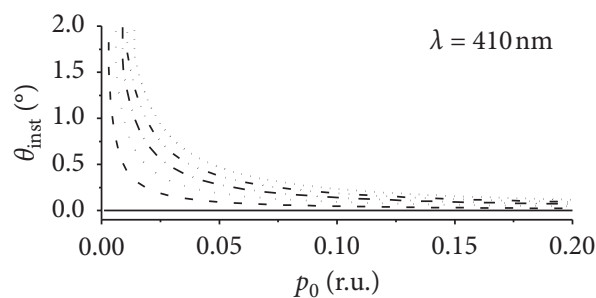

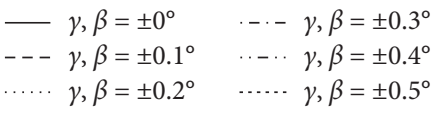

(b)

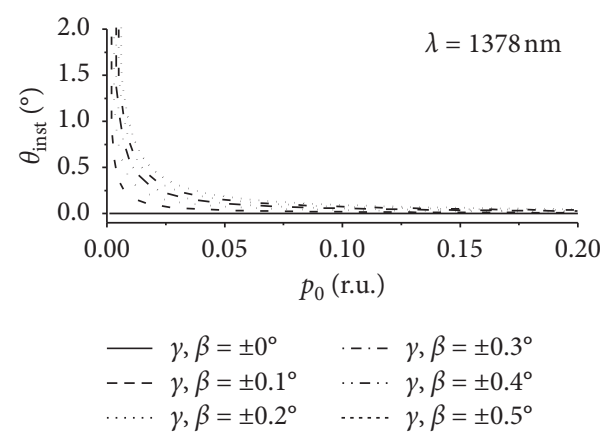

(d)

(c)

FIgURE 9: Continued. 


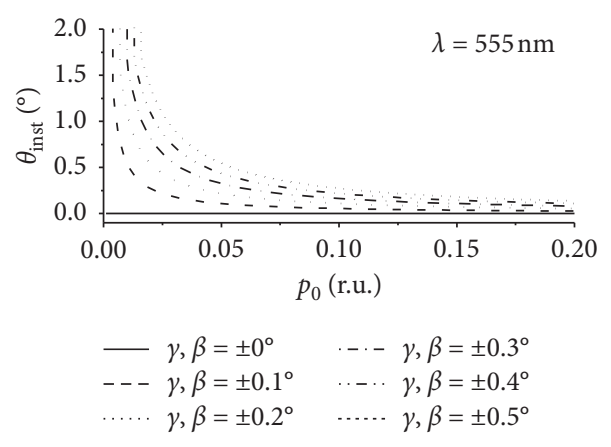

(e)

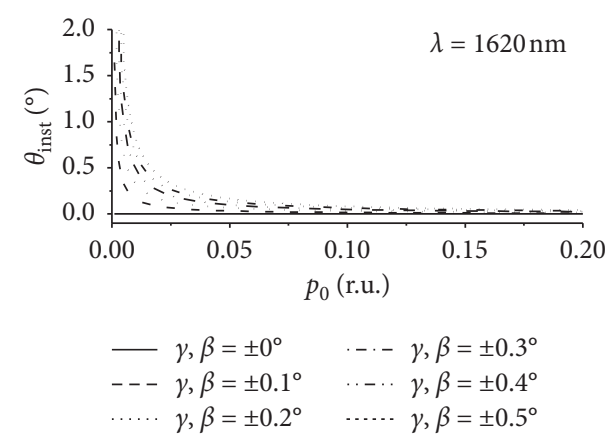

(f)

Figure 9: Dependence of the boundary value of the azimuth $\theta_{\text {inst }}$ of the instrumental polarization of the TSM on the mirror $M 2$ tilt around axes 1 and 2 (as shown in Figure 5) at degrees of linear polarization $p_{0}<0.2$.

$$
\begin{aligned}
& \beta \leq 15 \text { arc min }, \\
& \gamma \leq 15 \text { arc min },
\end{aligned}
$$

At higher values of errors in the position of TSM mirrors or if the increase in the accuracy of determining the angle of linear polarization of the input light is needed, it is necessary to calibrate the TSM according to the method described in [17].

4.2. The Procedure for Adjustment. To achieve an accuracy of 15 arcmin for the mirror installation in TSM, an appropriate method and optical bench were developed. Two certified standards of angles were used on the bench: a glass cube with surfaces of $90^{\circ}$ named a control optical element (COE) and a pentaprism guaranteeing to return the light beam at $90^{\circ}$. The angle tolerances of the glass cube and the pentaprism are 2 arcmin. The adjustment of the TSM includes the following steps:

Step 1 (illustrated in Figure 10): preassemble the TSM with the ability to rotate around its own axis. We use the axis that is a structural element of the electric motor and that is fixed during adjustment in the auxiliary mounting assembly. In input window 1, a framed COE is installed, which provides its tilt along the three axes. The COE is preliminarily mounted, such that its surface 1 is perpendicular to the TSM rotation axis. We control it as follows. The reflected beam from surface 1 on screen 1 must be stationary when rotating the TSM, i.e., $\mathrm{d} Y=0$. This guarantees that surface 1 is perpendicular to the TSM rotation axis with an error of \pm 2 arcmin. If this condition is not provided, adjust the COE using the screws, until $\mathrm{d} Y=0$. Next, we control that the beam from laser 1 passes through the center of window 1 and window 2 and put label $P 1$ on screen 2 .

Step 2 (illustrated in Figure 11): the pentaprism is placed in front of the TSM. It deflects laser beam 1 by $90^{\circ}$ on screen 2 . At this point, mark $P 2$ is made. The distance between labels $P 1$ and $P 2$ is determined in the projection on the $X$-axis.

Step 3 (illustrated in Figure 12): laser 2 is placed into the alignment system that directs the beam to the COE and

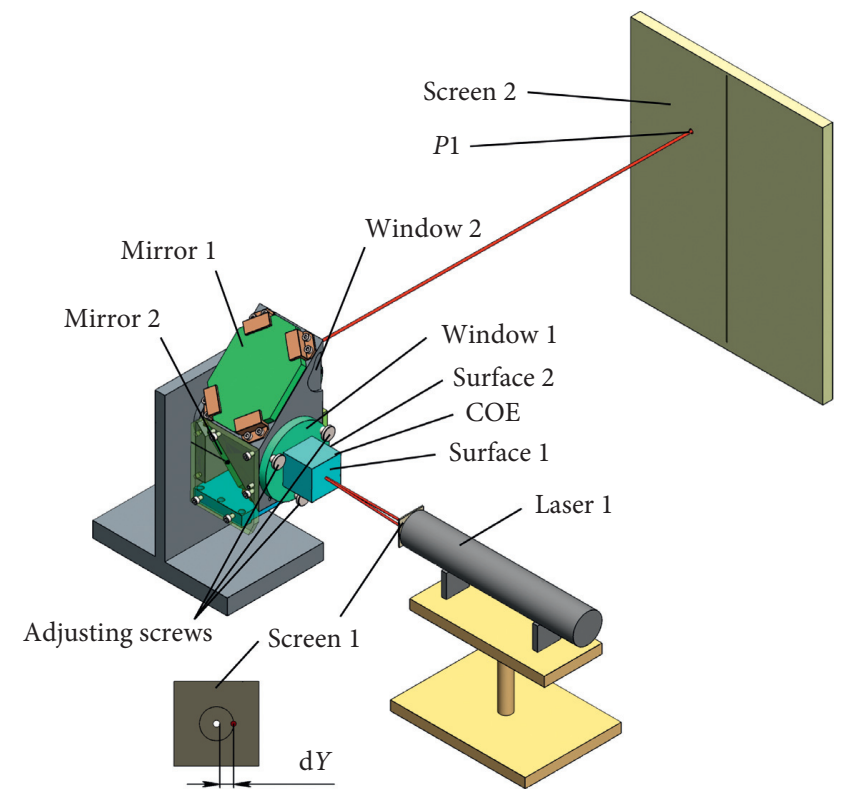

Figure 10: Adjustment of the TSM (Step 1).

the position of the reflected beam from the COE is fixed using label $P 3$. Label $P 3$ is selected arbitrarily. When we rotate the TSM by angle $90^{\circ}$, the second laser beam, reflected from the next face of the $\mathrm{COE}$, will create a label $P 4$. If label $P 4$ coincides with label $P 3$, it means that we have rotated the TSM by $90^{\circ} \pm 2$ arcmin.

Step 4 (illustrated in Figure 13): mirror 1 is removed, and the TSM is rotated at angle $90^{\circ}$. The correct position of the unit is controlled such that label $P 4$ coincides with label $P 3$ obtained in step 3. The distance between labels $P 2$ and $P 5$ in the projection on the $X$-axis is determined. If the segment $P 2-P 5$ is equal to the segment $P 1-P 2$, defined in step 2, mirror 2 is installed in the TSM at an angle of $90^{\circ}$. Otherwise, mirror 2 must be adjusted.

Step 5 (illustrated in Figure 14): place mirror 1 and rotate the TSM at an angle of $90^{\circ}$. The angle of rotation is controlled using a beam from laser 2, which is 


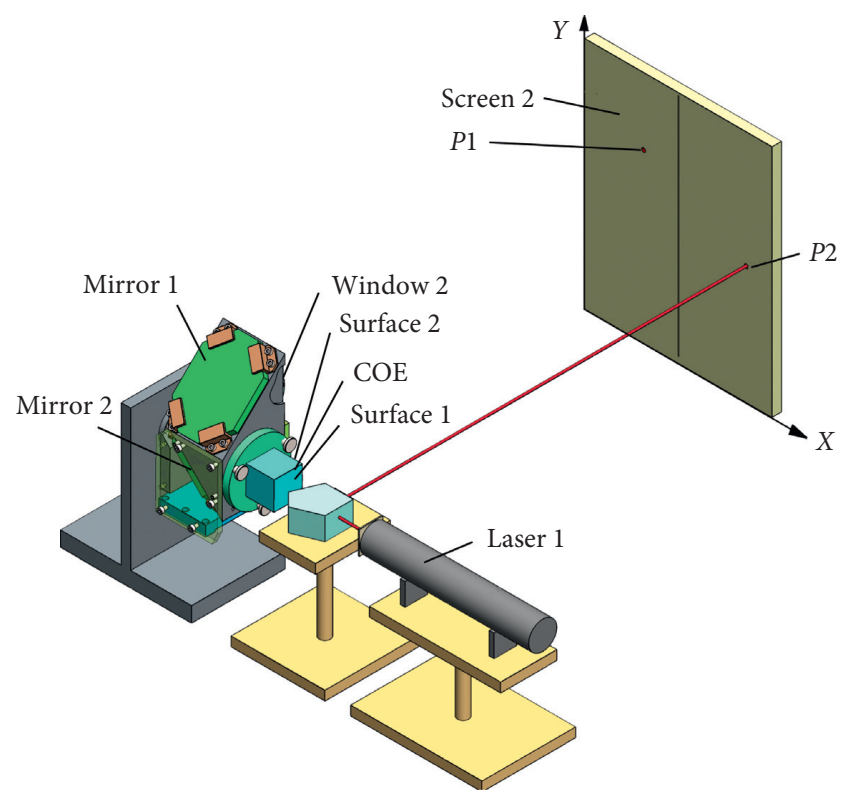

Figure 11: Adjustment of the TSM (Step 2)

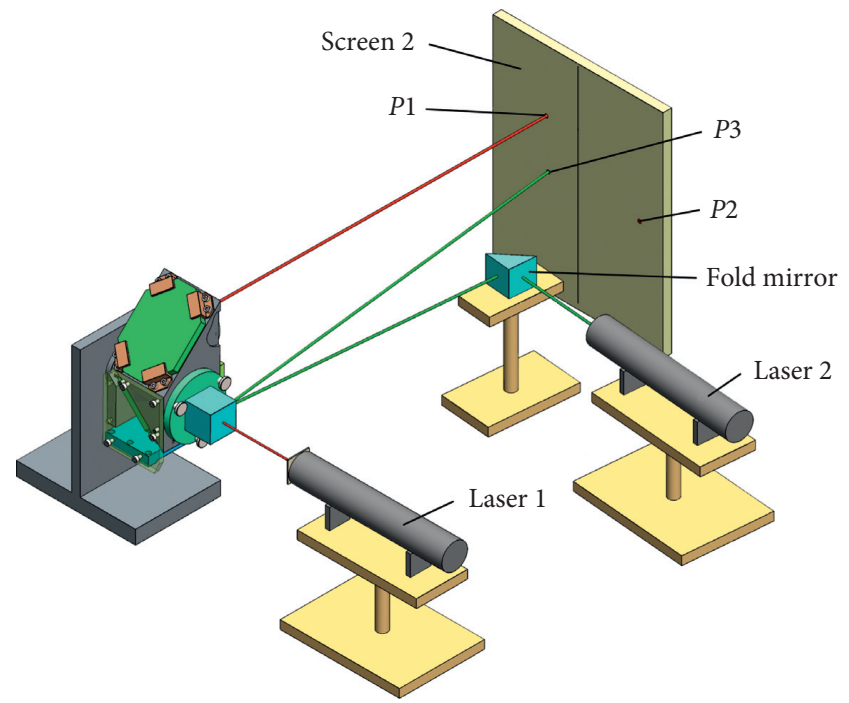

Figure 12: Adjustment of the TSM (Step 3).

reflected from the surface of the COE and must coincide with label $P 4$ on screen 2 .

We fix the position by label $P 6$ of the beam from laser 1 on screen 2 (Figure 14), which passed through the TSM. At the exact angle of mirror 2 relative to mirror 1 , equal to $90^{\circ}$, the spots from laser 1 must be placed on a single line along the $Y$-axis, which passes through label $P 5$, and must be at a distance of the base between mirror 1 and 2 centers from the specified label. If this condition is not satisfied, we adjust mirror 2 .

The distance between the TSM and screen 2 is 5 meters. The label position error on the screen and their alignment is $\leq 3 \mathrm{~mm}$, which gives an error of the viewing angle $<2$ arcmin. The aggregate error in the steps did not exceed 6 arcmin; accordingly, the angles of the mirrors were fixed with an

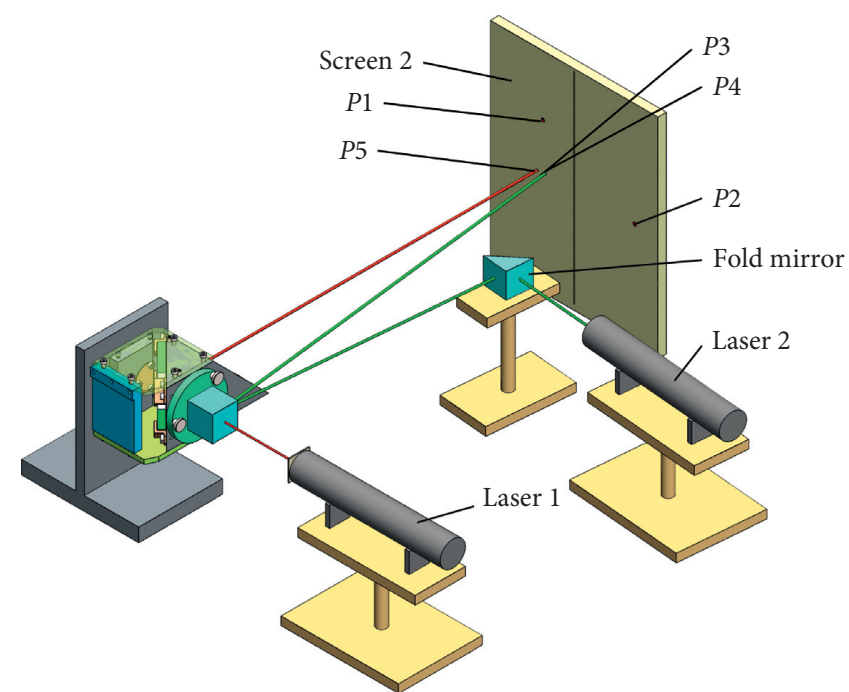

Figure 13: Adjustment of the TSM (Step 4).

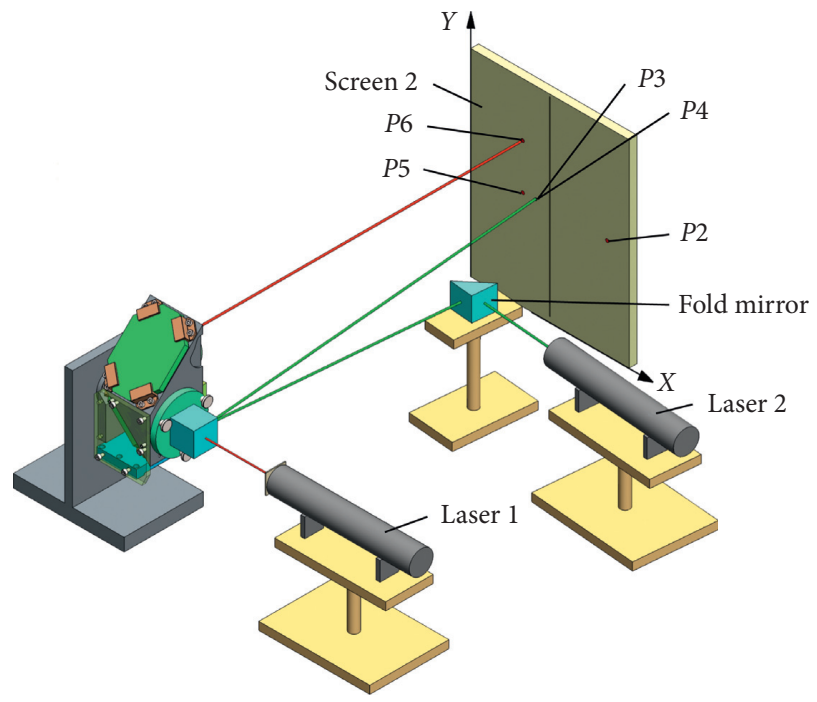

FIgUre 14: Adjustment of the TSM (Step 5).

error of $<3$ arcmin. Figure 15 shows a general view of a representation model of the SMA.

\section{Discussions}

It should be noted that modeling and analysis of polarization changes in optical systems with two or more crossed mirror elements have also been considered by other authors (see, for example, [18-20]). However, these works mainly consider the issue of modeling and analysis of polarization aberration in converging/diverging beams with a known spatial orientation of the mirrors, which, in addition, may have a different coating. Thus, the question of the influence of inaccuracies in adjustments of the mirrors in the mentioned optical systems on the instrumental polarization was not discussed directly. When developing the Research Scanning Polarimeter (RSP) [21] and Aerosol Polarimetry Sensor (APS) [22], which were taken as a basis at ScanPol, this issue 


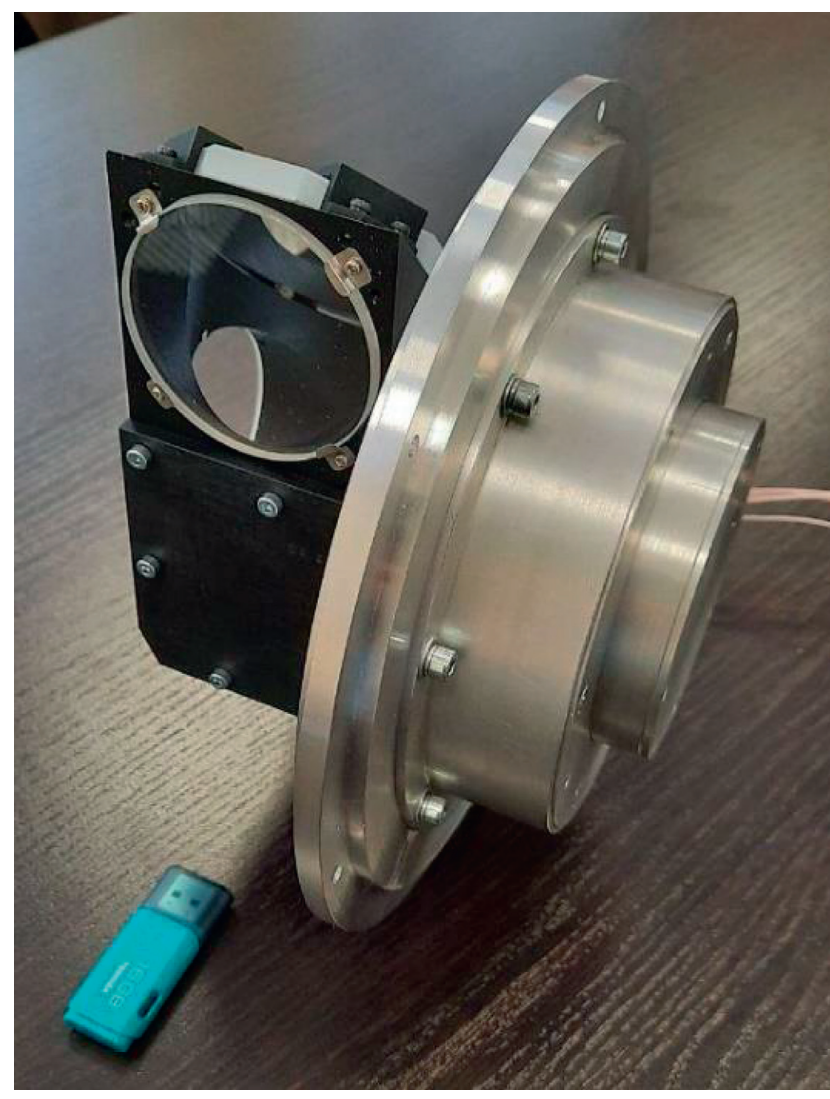

Figure 15: General view of the SMA.

was not discussed either. However, experimental studies have shown that, in the case of RSP, the instrumental polarization $p_{\text {inst }}$ did not exceed $0.1 \%$ [21], and in the case of APS $p_{\text {inst }}<0.05 \%$, in all bands except $\lambda=410 \mathrm{~nm}, p_{\text {inst }}<0.5 \%$ [23], which is probably due to the presence a certain resonant thickness for this wavelength range of $\mathrm{Al}_{2} \mathrm{O}_{3}$. At the same time, the iterative algorithm described in [24] can effectively compensate for instrumental polarization of $1 \%$. For the particulate observing scanning polarimeter (POSP) [25], whose design is also based on APS, the instrumental polarization was estimated at $0.5 \%$.

The mirror adjustment procedure, described in the presented work, allows setting the angles $\beta$ and $\gamma$ with an accuracy of $4-6 \operatorname{arcmin}\left(\leq 0.1^{\circ}\right)$. This theoretically allows keeping the instrumental polarization for ScanPol within $0.015 \%$ even in the worst case $\lambda=865 \mathrm{~nm}$, which is better than for APS case, although an acceptable value for ScanPol is $p_{\text {inst }} \leq 0.08 \%$, as mentioned above. Experimentally, the value of instrumental polarization for ScanPol will be estimated during the further ground calibration procedure.

When referring to instrumental polarization of ScanPol, we suppose instrumental polarization primarily of the mirror system, since the polarization imperfections of other ScanPol components, which are partially considered in [26], are effectively compensated by the calibration procedure described in [17].

\section{Summary}

We have developed a representational model of the ScanPol polarimeter scanning unit as an instrument of the AerosolUA space project for the investigation of the microphysical characteristics of the aerosol in the atmosphere. The developed electric motor with the compensator of the reactive moment and control system enables continuous scanning of the space under investigation with a constant speed during the movement of the satellite on an orbit and with a preservation of the invariability of its spatial position. Due to the use in the motor and compensator of contactless magnetoelectric disktype machines with a slotless stator, we have achieved the best energy, mass, size, and operational characteristics of the electric motor. The estimates of the dependence of the instrumental polarization of the TSM on the error of mirror adjustment have shown that one must be performed with an accuracy of less than 15 arcmin to achieve the error of determining the degree of linear polarization of light in all spectral ranges in ScanPol $\Delta p \leq 0.15 \%$. The developed procedure of assembling and adjusting the mirror system allowed us to install mirrors with a mutual arrangement error of less than 6 arcmin. This would minimize the contribution of the instrumental error to ScanPol measurements.

\section{Data Availability}

No data were used to support this study.

\section{Conflicts of Interest}

The authors declare no conflicts of interest.

\section{Acknowledgments}

The authors extend special thanks to Olexandr Antonov from the Institute of Electrodynamics of the National Academy of Science of Ukraine. Unfortunately, he passed away. Dr. Antonov made a significant contribution to the research in the manuscript. This research was supported by the National Academy of Science of Ukraine under Target Comprehensive Program for Space Research for 2018-2022.

\section{References}

[1] O. Dubovik, Z. Li, M. I. Mishchenko et al., "Polarimetric remote sensing of atmospheric aerosols: instruments, methodologies, results, and perspectives," Journal of Quantitative Spectroscopy and Radiative Transfer, vol. 224, pp. 474-511, 2019.

[2] G. Milinevsky, Y. Yatskiv, O. Degtyaryov et al., "Remote sensing of aerosol in the terrestrial atmosphere from space: new missions," Advances in Astronomy and Space Physics, vol. 5, pp. 11-16, 2015.

[3] G. Milinevsky, Y. Yatskiv, O. Degtyaryov et al., "New satellite project Aerosol-UA: remote sensing of aerosols in the terrestrial atmosphere," Acta Astronautica, vol. 123, pp. 292-300, 2016. 
[4] I. I. Syniavskyi, S. Yu, M. G. Ivanov, G. P. Sosonkin, and M. G Koshman, "Multispectral imager-polarimeter of the "AEROSOL-UA" space project," Space Science and Technology, vol. 24, no. 3, pp. 23-32, 2018.

[5] I. I. Syniavskyi, G. P. Milinevsky, S. Yu et al., "Methodology, hardware implementation, and validation of satellite remote sensing of atmospheric aerosols: first results of the AerosolUA space experiment development," Space Science and Technology, vol. 21, no. 3, pp. 9-17, 2015.

[6] A. E. Antonov, K. P. Akinin, and V. G. Kireev, "Creation features of electromechanical system for nanosatellite orientation on the basis of brushless magnetoelectric motor," Tekhnichna Elektrodynamika, vol. 4, pp. 36-40, 2017.

[7] A. Antonov, K. Akinin, and V. Kireev, "Compersation of the reaction moment in electric drive on board systems," Tekhnichna Elektrodynamika, vol. 3, pp. 66-73, 2018.

[8] V. G. Kireyev, "Construction of the optimal brushless motor of disk type with permanent magnets," Tekhnichna Elektrodynamika, vol. 6, pp. 38-43, 2012.

[9] R. A. Chipman, W.-S. T. Lam, and G. Young, Polarized Light and Optical Systems, Taylor \& Francis CRC Press, Boca Raton, FL, USA, 2018.

[10] G. van Harten, F. Snik, and C. U. Keller, "Polarization Properties of Real Aluminum Mirrors, I. Influence of the Aluminum oxide layer," Publications of the Astronomical Society of the Pacific, vol. 121, pp. 377-383, 2009.

[11] K. M. McPeak, S. V. Jayanti, S. J. P. Kress et al., "Plasmonic films can easily be better: rules and recipes," ACS Photonics, vol. 2, no. 3, pp. 326-333, 2015.

[12] A. D. Rakić, "Algorithm for the determination of intrinsic optical constants of metal films: application to aluminum," Applied Optics, vol. 34, no. 22, pp. 4755-4767, 1995.

[13] A. Lehmuskero, M. Kuittinen, and P. Vahimaa, "Refractive index and extinction coefficient dependence of thin $\mathrm{Al}$ and $\mathrm{Ir}$ films on deposition technique and thickness," Optics Express, vol. 17, pp. 10744-10752, 2007.

[14] F. Cheng, P.-H. Su, J. Choi, S. Gwo, X. Li, and C.-K. Shih, "Epitaxial growth of atomically smooth aluminum on silicon and its intrinsic optical properties," ACS Nano, vol. 10, pp. 9852-9860, 2016.

[15] https://www.filmetrics.com/refractive-index-database/Al/ Aluminium.

[16] https://refractiveindex.info/?shelf=main\&book=Al\&page= McPeak.

[17] G. Milinevsky, Y. Oberemok, I. Syniavskyi et al., "Calibration model of polarimeters on board the Aerosol-UA space mission," Journal of Quantitative Spectroscopy \& Radiative Transfer, vol. 229, pp. 92-105, 2019.

[18] D. G. Crandall and R. A. Chipman, "Polarization aberrations of crossed folding mirrors," Proceedings of the SPIE, vol. 2537, pp. 83-93, 1995.

[19] W. S. T. Lam and R. Chipman, "Balancing polarization aberrations in crossed fold mirrors," Applied Optics, vol. 54, no. 11, pp. 3236-3245, 2015.

[20] D. M. Harrington and R. Stacey, "Polarization modeling and predictions for Daniel K. Inouye Solar Telescope part 1: telescope and example instrument configurations," Journal of Astronomical Telescopes, Instruments, and Systems, vol. 3, no. 1, p. 18002, 2017.

[21] B. Cairns, E. E. Russell, and L. D. Travis, "Research scanning polarimeter: calibration and ground-based measurements," Proceedings of the SPIE, vol. 3754, pp. 186-196, 1999.
[22] R. J. Peralta, C. Nardell, B. Cairns et al., "Aerosol polarimetry sensor for the glory mission," Proceedings of the SPIE, vol. 17, p. 6786, 2007.

[23] B. Cairns, M. Mishchenko, H. Maring, B. Fafaul, and S. Pszcolka, "Accurate monitoring of terrestrial aerosols and total solar irradiance: the NASA Glory mission," Proceedings of the SPIE, vol. 7826, p. 78260, 2010.

[24] B. Cairns and I. Geogdzhayev, "GSFC 421.7-70-03 aerosol polarimetry sensor algorithm theoretic basis document," 2010, http://glory.giss.nasa.gov/aps/docs/APS_ATBD_CALI BRATE_CCB.pdf.

[25] H. C. Yang, B. Y. Yang, M. X. Song, P. Zou, X. B. Sun, and J. Hong, "Onboard Polarimetric Calibration Methods of Spaceborne Scanning Polarimeter," Chinese Journal of Lasers, vol. 45, no. 11, Article ID 1110002, 2018.

[26] I. Syniavskyi, Y. Oberemok, Y. Ivanov, and M. Sosonkin, "Multispectral polarization state analyzer of scanning polarimeter ScanPol," International Journal of Optics, vol. 2020, Article ID 1695658, 15 pages, 2020. 